\title{
Diabetic and Non-Diabetic Gastroparesis: A Retrospective Comparative Outcome Study From the Nationwide Inpatient Sample
}

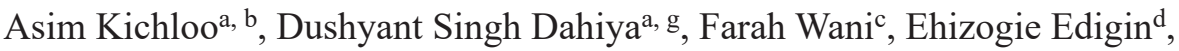 \\ Jagmeet Singhe, Micheal Albosta ${ }^{a}$, Asad Mehboob ${ }^{f}$, Hafeez Shaka ${ }^{d}$
}

\begin{abstract}
Background: Gastroparesis (GP), meaning "gastric palsy", is a chronic medical condition characterized by delayed or absent gastric emptying in the absence of mechanical obstruction. The primary objective of this study was to determine the patient-specific outcomes and the burden of GP on the US healthcare system.

Methods: This was a population-based, retrospective study designed to analyze data available from the Nationwide Inpatient Sample (NIS) for 2016 and 2017. Using the International Classification of Diseases, Tenth Revision (ICD-10) codes, we identified adult (18 years or older) hospitalizations with a principal diagnosis of GP. The study sample was further classified into two distinct groups based on the presence or absence of GP, namely diabetic GP (DGP) and non-diabetic GP (NDGP). The primary outcome of our study was inpatient mortality secondary to GP while the secondary outcomes included the odds of system-based complications and the burden of the disease on the United States healthcare system.
\end{abstract}

Results: We identified 99,695 adult ( $\geq 18$ years) hospitalizations with a principal discharge diagnosis of GP in 2016 and 2017. Of these patients, $78.1 \%(77,885)$ had DGP and $21.9 \%(21,810)$ were classified in the NDGP group. We noted a higher proportion of women $(79.3 \%$ vs. $63.4 \%, \mathrm{P}<0.001)$, patients with a history of smoking $(35.8 \%$ vs.

Manuscript submitted January 17, 2021, accepted February 11, 2021

Published online February 19, 2021

aDepartment of Internal Medicine, Central Michigan University, College of Medicine, Saginaw, MI, USA

bDepartment of Internal Medicine, Samaritan Medical Center, Watertown, NY, USA

'Department of Family Medicine, Samaritan Medical Center, Watertown, NY, USA

${ }^{\mathrm{d}}$ Department of Internal Medicine, Geisinger Commonwealth School of Medicine, Scranton, PA, USA

eDepartment of Internal Medicine, John H. Stronger Jr. Hospital of Cook County, Chicago, IL, USA

fDepartment of Gastroenterology, Covenant Healthcare, Saginaw, MI, USA 'Corresponding Author: Dushyant Singh Dahiya, Department of Internal Medicine, Central Michigan University, 1000 Houghton Avenue, Saginaw, MI 48602, USA. Email: dush.dahiya@gmail.com

doi: https://doi.org/10.14740/gr1364
$31.7 \%, \mathrm{P}<0.001)$ and chronic obstructive pulmonary disease $(10.7 \%$ vs. $7.6 \%, \mathrm{P}<0.001)$ in the NDGP group. After adjusting for common confounders excluding age, the inpatient mortality for GP was found to be $0.25 \%$. Furthermore, after adjustments were made for the Charlson comorbidity index (CCI), we noted higher odds of mortality in the NDGP group ( $0.30 \%$ vs. $0.23 \%$, adjusted odds ratio (aOR): $3.18,95 \%$ confidence interval $(\mathrm{CI}): 1.75-5.79, \mathrm{P}<0.001)$ compared to the DGP group. Additionally, patients with NDGP had a higher mean length of stay ( 5 vs. 4.1 days, $\mathrm{P}<0.001$ ) and higher mean total hospital charge $(\$ 44,100$ vs. $\$ 35,500, \mathrm{P}<0.001)$ compared to those with DGP. The NDGP group also had higher odds of sepsis, deep vein thrombosis (DVT) and pulmonary embolism (PE); however, the odds of developing acute kidney injury (AKI) were lower than that of the DGP group.

Conclusions: The inpatient mortality for GP was found to be $0.25 \%$. After adjusting for CCI, patients with NDGP had higher odds of inpatient mortality compared to the DGP group. Additionally, patients with NDGP were associated with a longer length of hospital stay, mean total healthcare cost and higher odds of complications such as sepsis, DVT and PE during the hospitalization.

Keywords: Non-diabetic gastroparesis; Diabetic gastroparesis; Outcome; Mortality; Nationwide inpatient sample; Complications; Total hospital cost; Mean length of stay

\section{Introduction}

Gastroparesis (GP) is a chronic medical disorder characterized by delayed or absent gastric emptying in the absence of a mechanical obstruction [1]. As the association of diabetes and GP is most frequently encountered in clinical practice, majority of the studies conducted have explored this relationship [2, 3]. As per literature, the exact incidence and prevalence of GP in the general population is unknown [4]. However, the most common cause of GP is believed to idiopathic, seen in $35 \%$ of the patients, followed by diabetes mellitus which may be present in up to $29 \%$ of the patient population [5]. Although there are numerous studies on GP, there is limited data on the mortality of GP in an inpatient setting. Hence, in this study, we use the Nationwide Inpatient Sample (NIS) database to determine patient-specific outcomes and factors associated with GP in 
both diabetic and non-diabetic hospitalizations while comparing the two groups. We also aimed to estimate the inpatient mortality rates for GP, measure the disease burden on the US healthcare system in terms of cost of management and length of hospital stay, and determine the odds of developing certain system-based complications in an inpatient setting. Through this analysis, we hope to bridge the gap in knowledge that currently exists for hospitalizations due to GP.

\section{Materials and Methods}

\section{Design and data source}

This was a retrospective cohort study involving all adult $(\geq$ 18 years) hospitalizations for a principal diagnosis of GP in the US between January 1, 2016 and December 31, 2017. The data were sourced from the NIS database, which is a publicly available database of inpatient hospital stays derived from the billing data submitted by hospitals to statewide data organizations across the US, thereby covering more than $97 \%$ of the US population $[6,7]$. It approximates a $20 \%$ stratified sample of discharges from US community hospitals, excluding rehabilitation and long-term acute care hospitals. This dataset is weighted to obtain national estimates [6]. The 2016 and 2017 databases were entirely coded using the International Classification of Diseases, Tenth Revision, Clinical Modification/Procedure Coding System (ICD-10-CM/PCS). In NIS, diagnoses are divided into two separate categories, namely principal diagnosis, and secondary diagnosis. The principal diagnosis was the main ICD-10 code for the hospitalization and the secondary diagnosis was any ICD-10 code other than the principal diagnosis.

\section{Study population}

The NIS database was queried for all adult ( $\geq 18$ years) hospitalizations with a primary diagnosis of GP for 2016 and 2017. The NIS database uses the ICD coding system to classify and store data for inpatient hospitalizations. We used the ICD-10 codes E08.43, E09.43, E10.43, E11.43, and E13.43 as they correlate with diabetic GP (DGP). Additionally, patients with unspecified GP (ICD-10 code K31.84) with a secondary diagnosis of diabetes mellitus were included in the DGP group and those with unspecified GP without a secondary diagnosis of diabetes mellitus were included in the non-diabetic GP (NDGP) cohort.

\section{Outcome measures}

The primary outcome of the study was a comparison of inpatient mortality between the DGP and NDGP groups. Secondary outcomes included development of sepsis, acute kidney injury (AKI), deep vein thrombosis (DVT), pulmonary embolism (PE), mean length of stay (LOS) in the hospital and mean total hospital charges (THC).

\section{Statistical analysis}

We analyzed the data using Stata ${ }^{\circledR}$ version 16 software (StataCorp, Texas, USA). All the analyses were conducted using weighted samples for national estimates in adjunct with Healthcare Cost and Utilization Project (HCUP) regulations for using the NIS database. Comorbidities were calculated as proportions of the cohort and Chi-squared test was used to compare these characteristics between the DGP and NDGP subgroups. Multivariate regression analysis was utilized to adjust for possible confounders while calculating the primary and secondary outcomes, which were obtained from literature review. All P values were two-sided, with 0.05 as the threshold for statistical significance.

\section{Ethical considerations}

The NIS database lacks patient identifiers. In 2012, NIS also removed state level and hospital identifiers. This has enhanced patient privacy, protection, and anonymity. Hence, this study was exempt from Institutional Review Board (IRB) approval.

\section{Results}

\section{Patient characteristics}

For 2016 and 2017, NIS contained over 71 million weighted hospital discharges out of which 99,695 hospitalizations were included in the study. Of these hospitalizations, 78.1\% $(77,885)$ of the patients had DGP and $21.9 \%(21,810)$ were included in the NDGP group. We did not observe a statistically significant difference in the mean age for DGP and NDGP. Compared to patients with DGP, we noted that the NDGP group had a higher proportion of women $(79.3 \%$ vs. $63.4 \%, \mathrm{P}<0.001)$, history of smoking (35.8\% vs. $31.7 \%, \mathrm{P}<0.001)$ and patients with chronic obstructive pulmonary disease $(10.7 \%$ vs. $7.6 \%, \mathrm{P}<0.001)$; however, the DGP group had higher proportion of patients with hypertension ( $40.5 \%$ vs. $32.8 \%, \mathrm{P}<0.001)$, obesity $(18.6 \%$ vs. $13.7 \%, \mathrm{P}<0.001)$, congestive heart failure $(13.0 \%$ vs. $4.9 \%, \mathrm{P}<$ $0.001)$ and chronic kidney disease $(32.1 \%$ vs. $6.6 \%, \mathrm{P}<0.001)$. Furthermore, statistically significant racial differences were also noted between the two subgroups with NDGP being more common in the Whites $(69.8 \%$ vs. $40.2 \%, \mathrm{P}<0.001)$ and DGP more frequently seen in Black (35.4\% vs. $17.4 \%, \mathrm{P}<0.001)$ and Hispanic $(14.2 \%$ vs. $6.7 \%, \mathrm{P}<0.001)$ populations. The patient and hospital characteristics are summarized in Table 1.

\section{Primary outcome: inpatient mortality}

The inpatient mortality for GP was found to be $0.25 \%$. After adjusting for CCI, patients in the NDGP group had higher odds of inpatient mortality $(0.30 \%$ vs. $0.23 \%$, adjusted odds ratio (aOR): $3.18,95 \%$ confidence interval $(\mathrm{CI}): 1.75-5.79, \mathrm{P}<$ 
Table 1. Patient and Hospital Characteristics of Hospitalizations for Gastroparesis

\begin{tabular}{|c|c|c|c|}
\hline Variable & Diabetic gastroparesis (\%) & Non-diabetic gastroparesis (\%) & P value \\
\hline Sample size & $77,885(78.1 \%)$ & $21,810(21.9 \%)$ & \\
\hline \multicolumn{4}{|l|}{ Patient characteristics } \\
\hline Age, mean & 47.3 & 47.4 & 0.676 \\
\hline Women & 63.4 & 79.3 & $<0.001$ \\
\hline Racial distribution & & & $<0.001$ \\
\hline White & 40.2 & 69.8 & \\
\hline Hispanic & 14.2 & 6.7 & \\
\hline Others & 7.2 & 6.1 & \\
\hline Insurance type & & & $<0.001$ \\
\hline Medicaid & 43.8 & 40.2 & \\
\hline Medicare & 28.5 & 22.0 & \\
\hline 0 & 0.1 & 52.3 & \\
\hline 1 & 1.5 & 25.3 & \\
\hline 2 & 39.3 & 11.4 & \\
\hline$\geq 3$ & 59.1 & 11.0 & \\
\hline Median annual income in patient's zip code $\left(\mathrm{USD}^{\mathrm{a}}\right)$ & & & $<0.001$ \\
\hline $1-43,999$ & 42.6 & 31.2 & \\
\hline $44,000-55,999$ & 25.5 & 27.6 & \\
\hline $56,000-73,999$ & 20.2 & 23.3 & \\
\hline$\geq 74,000$ & 11.7 & 17.9 & \\
\hline \multicolumn{4}{|l|}{ Comorbidities } \\
\hline COPD & 7.6 & 10.7 & $<0.001$ \\
\hline Electrolyte derangements & 38.8 & 36.6 & 0.020 \\
\hline \multicolumn{4}{|l|}{ Hospital characteristics } \\
\hline Hospital region & & & 0.026 \\
\hline Northeast & 15.0 & 16.3 & \\
\hline Midwest & 18.8 & 17.9 & \\
\hline South & 48.3 & 50.1 & \\
\hline West & 17.9 & 15.7 & \\
\hline Hospital bed size & & & 0.201 \\
\hline Small & 18.4 & 16.7 & \\
\hline Medium & 31.2 & 31.7 & \\
\hline Large & 50.4 & 51.6 & \\
\hline Urban location & 92.8 & 93.5 & 0.121 \\
\hline Teaching hospital & 68.0 & 70.3 & 0.019 \\
\hline
\end{tabular}

a2017. CHF: congestive heart failure; CKD: chronic kidney disease; COPD: chronic obstructive pulmonary disease; CVA: cerebrovascular accident; IHD: ischemic heart disease. 
Table 2. Clinical Outcomes in Patients With Gastroparesis

\begin{tabular}{|c|c|c|c|c|}
\hline Outcome & DGP (\%) & NDGP (\%) & $\operatorname{aOR}(95 \% \mathrm{CI})$ & P value* \\
\hline \multicolumn{5}{|l|}{ Primary outcome } \\
\hline In hospital mortality & 0.23 & 0.30 & $3.18(1.75-5.79)$ & $<0.001^{*}$ \\
\hline Mean length of stay & 4.1 & 5.0 & $1.4(1.2-1.6)^{\mathrm{a}}$ & $<0.001^{*}$ \\
\hline Mean total hospital charges (USD) & 35,500 & 44,100 & $14,000(11,500-16,500)^{\mathrm{a}}$ & $<0.001^{*}$ \\
\hline AKI & 20.60 & 8.09 & $0.63(0.55-0.72)$ & $<0.001^{*}$ \\
\hline DVT & 0.91 & 1.05 & $1.91(1.27-2.87)$ & $0.002 *$ \\
\hline $\mathrm{PE}$ & 0.19 & 0.37 & $2.28(1.03-5.02)$ & $0.041 *$ \\
\hline
\end{tabular}

*Statistically significant. aAdjusted mean difference. aOR: adjusted odds ratio; Cl: confidence interval; AKI: acute kidney injury; DGP: diabetic gastroparesis; DVT: deep venous thrombosis; NDGP: non-diabetic gastroparesis; PE: pulmonary embolism.

0.001) compared to those with DGP.

\section{Secondary outcomes}

Patients with NDGP had a higher mean LOS at 5 days (1.4, $95 \% \mathrm{CI}: 1.2-1.6, \mathrm{P}<0.001)$ and higher mean THC at $\$ 44,100$ (14,000, 95\% CI: $11,500-16,500, \mathrm{P}<0.001)$ compared to those with DGP at 4.1 days and $\$ 35,500$, respectively. Additionally, higher odds of sepsis (aOR: 1.70, 95\% CI: $1.02-2.84, \mathrm{P}=$ 0.043), DVT (aOR: 1.91, 95\% CI: $1.27-2.87, \mathrm{P}=0.002)$ and PE (aOR: 2.28, 95\% CI: 1.03 - 5.02, P = 0.041) were observed in the NDGP group compared to those with DGP. However, NDGP patients had lower odds of developing AKI (aOR: 0.63, 95\% CI: $0.55-0.72, \mathrm{P}<0.001)$ compared to patients with DGP. The secondary outcomes are summarized in Table 2.

\section{Discussion}

GP was first described in the mid-1900s; but over the last few decades, it has grabbed the interest of investigators [8,9]. A deeper understanding of the etiology and pathophysiological mechanisms of the disease helps in the development of targeted therapeutic strategies. GP is a relatively under-investigated disease pathology not only due to a significant overlap of clinical features with functional dyspepsia, but also because most epidemiological studies primarily investigate the association of GP and diabetes mellitus [2, 3]. Furthermore, a minor fraction of patients with function dyspepsia may have delayed gastric emptying times blurring the distinction between idiopathic GP and functional dyspepsia [10]. Hence, there is consensus among physicians that GP is an underdiagnosed disease entity, and its true impact on the general population may still be unknown [4]. GP impacts the overall quality of life of patients, and also places significant burden on the healthcare system both in terms of hospital cost and the resources needed to treat these patients. Hence, this study was designed to estimate the epidemiology, identify possible associations, and adverse outcomes of GP hospitalizations.

The true incidence and prevalence of GP are not known due to difficulties in undertaking large, controlled populationbased studies. Literature describes only two large prospective studies on GP till date, both of which relied on data collection from hospitalizations rather than the general population [11]. One of these studies, namely the Rochester Epidemiology Project, was a large community-based study conducted in Olmsted County of Minnesota [12]. It reported an age-adjusted prevalence of 9.6 per 100,000 in men and 37.8 per 100,000 in women for GP [12]. Rey et al further utilized this data to report a prevalence rate of 24.2 per 100,000 persons and an incidence rate of 6.3 per 100,000 persons per year for GP [13]. From 1997 to 2013, hospitalizations secondary to GP have increased by $300 \%$ and can be attributed to either a true increase in prevalence or overdiagnosis of the disease pathology [11]. In the study period, we report 99,695 hospitalizations for GP, out of which $77,885(78.1 \%)$ had DGP while $21,810(21.9 \%)$ were classified in the NDGP group. Although there was no statistical difference in the mean age for both groups, we report a higher percentage of women in the NDGP group. These findings were in line with current literature. Furthermore, we noted racial difference between the two groups. GP secondary to a diabetic etiology was more frequent in Blacks and Hispanics, whereas NDGP was more commonly seen in Whites. This could be attributed to a higher prevalence of diabetes mellitus in the racial/ethnic minorities such as Blacks and Hispanics as compared to Whites [14]. Additionally, it has been reported that racial/ethnic minorities are more likely to experience complications of diabetes compared to other populations.

\section{Classification of GP}

GP can be subdivided into numerous categories based on the underlying pathophysiology and associations with other diseases. Identification of the underlying pathophysiological mechanism leading to GP may help direct treatment in cases, where withdrawal of the inciting agent (medication-induced GP) or correction of the underlying disease process may lead to resolution of symptoms. Overall, the two most common etiologies implicated for GP includes idiopathic causes and dia- 
betes mellitus. The classification of GP is as follows.

\section{Idiopathic GP}

Although there is paucity of data investigating non-diabetic causes of GP, idiopathic GP is believed to be the most common subtype of GP. As per literature, idiopathic causes may constitute as high as $35 \%$ of all patients with GP [5].

\section{$D G P$}

Diabetes mellitus is a group of chronic medical conditions characterized by hyperglycemia secondary to insufficient insulin secretion, resistance to action of insulin on tissues, or both [15]. Gastrointestinal complications of diabetes, usually secondary to autonomic dysfunction or abnormal intrinsic nervous system function, are seen in patients who have had persistent disease, typically of more than 5 years' duration [16]. Over the years, the association of diabetes and GP has been extensively studied. In a large, single center cohort study, it was estimated that about one-third $(29 \%)$ of the patients with GP had underlying diabetes $[17,18]$. However, this study had a sampling bias as the study sample included only hospitalized patients. In the general population, this rate is expected to be much lower $[17,18]$. Similarly, for our study, the study sample was obtained from a large publicly available inpatient database. Of all the hospitalizations for GP, we report that $78.1 \%$ had associated diabetes mellitus. This may be because hospitals have a greater influx of patients with diabetes and are therefore more likely to establish a diagnosis of DGP compared to its actual prevalence in the general population.

\section{Post-surgical GP (PSGP)}

As per literature, PSGP may be seen in about $13 \%$ of the patients with GP [5]. Injury to the vagus nerve following gastric or thoracic surgical intervention (i.e., hiatal hernia repairs, organ transplantation, gastrectomy, fundoplication, and Rouxen-Y gastric bypass surgery, etc.) is commonly implicated [19]. Additionally, the surgical intervention itself may lead to satiety and delayed emptying times such as that seen after Roux-en-Y anastomosis.

\section{Post-infectious GP}

A subset of patients may report sudden onset GP after an infection. It is frequently seen after viral infections (post-viral GP (PVGP)), and patients often report a viral prodrome before the onset of clinical symptoms. Studies report that PVGP may be seen in up to $8.2 \%$ of patients with GP [20]. Viral pathogens most commonly implicated in PVGP include rotavirus and Norwalk virus [21, 22]. Other viral infections such as Cytomegalovirus (CMV), Epstein-Barr Virus (EBV) and Varicella-Zoster Virus (VZV) may also be involved [23, 24]. PVGP is often self-limiting with excellent prognosis and gradually improves over 1 year [ 21 , 25]. However, persistent symptoms may be seen in CMV, EBV or VZV infections secondary to severe dysautonomia $[24,26]$.

\section{Medication-induced GP}

Certain medications are known to delay gastric emptying through their action on numerous receptors. Medication-induced GP may be seen with narcotics, cyclosporine, phenothiazines, dopamine agonists, octreotide, alpha-2-adrenergic agonists such as clonidine, tricyclic antidepressants, calcium channel blockers, GLP-1 agonists (exenatide) or analogues (liraglutide), lithium and progesterone [27]. Establishing a diagnosis of medication-induced GP requires a thorough history and high degree of suspicion. It has excellent prognosis and improves after withdrawal of the inciting agent.

\section{GP associated with neurological conditions}

Central nervous system (CNS) disorders and dysautonomia can hinder gastrointestinal motility via their effect on the sympathetic or parasympathetic nervous system [28]. The vagus nerve and the thoracic spinal sympathetic outflow may be involved in patients with Parkinson's disease, multiple sclerosis, brainstem stroke, brainstem tumors, diabetic neuropathy, amyloid neuropathy or primary dysautonomia $[27,29]$. Furthermore, involvement of the myenteric neuronal plexus may be seen in patients with diffuse neurological disorders such as diabetes, human immunodeficiency virus (HIV)/acquired immunodeficiency syndrome (AIDS), and Parkinson's disease [29, 30]. Of all the neurological disorders, Parkinson's disease is closely associated with GP and may be seen in about $7.5 \%$ of all patients with GP [20].

\section{Autoimmune GP}

Autoimmune disease is characterised by the presence of autoantibodies and may present with a wide spectrum of clinical manifestations secondary to autoimmune dysautonomia [31]. It may be of idiopathic origin or seen in association with anatomically remote neoplasms, the most common being small cell lung carcinoma [32]. Autoimmune GP may also present with clinical features of slow intestinal and colonic transit times, or uncoordinated pelvic floor muscle contractions along with GP [32]. Although numerous antibodies have been studied, high antibody titres of anti-glutamic acid decarboxylase (GAD) antibodies have been noted to have a close association with autoimmune dysautonomia [33]. Furthermore, literature emphasizes the benefit of plasmapheresis in these patients [34].

\section{Miscellaneous causes}

Other causes of GP such as mesenteric ischemia, connective tissue disorders, collagen vascular disorders, scleroderma, amyloidosis, endocrine disorders (hypothyroidism) and renal 
insufficiency have also been described [35, 36].

\section{Associations and establishing a diagnosis of GP}

In our study, we analyzed specific associations of GP. Compared to DGP hospitalizations, the NDGP group had a higher proportion of patients with a history of smoking and chronic obstructive pulmonary disease (COPD) despite making up less than one-third of the study sample. The exact reason of this distribution is currently unknown. Additionally, we noted a higher proportion of hypertension, obesity, congestive heart failure and chronic kidney disease in DGP hospitalizations compared to NDGP hospitalizations. Hence, we strongly advocate for the need of additional large, prospective cohort studies to further explore these associations and establish causality.

GP usually presents with a wide spectrum of clinical signs and symptoms along with features of the underlying etiology. Patients with idiopathic GP most commonly report symptoms of early satiety, fullness after meals and abdominal pain [37]. However, patients with DGP may have severe retching and vomiting as the predominant symptom [37]. Some common symptoms of GP $[3,38,39]$ include nausea (93\%), vomiting $(64-84 \%)$; abdominal pain (46-90\%); bloating (mild - $73 \%$ and severe - $41 \%$ ); early satiety; post-prandial fullness and loss of weight and apatite.

Abdominal examination may be unremarkable or reveal epigastric distention. A suction splash or gastric splash (sloshing sound heard on abdominal auscultation during sudden movements) may also be noted and reflects the presence of fluids in the stomach secondary to the delayed or absent gastric emptying. Abdominal tenderness may also be reported in some patients. However, abdominal guarding and rigidity are usually absent.

Initial workup to establish a diagnosis of GP involves a thorough history and physical examination. GP should be suspected in patients presenting with nausea, vomiting, abdominal pain, bloating and post-prandial fullness [40]. Additionally, physicians should be on the lookout for common associations of GP. However, most cases of GP may be idiopathic; hence, identification of the underlying etiology may not always be possible. Upper gastrointestinal endoscopy should be performed in patients with features of delayed gastric emptying and abdominal pain for an obvious cause [27]. Furthermore, radiological imaging modalities such as computed tomography (CT) and magnetic resonance imaging (MRI) are needed to rule out mechanical obstruction [27]. In patients without mechanical obstruction, gastric emptying study (GES) is the next best step. It is cost effective, widely available, and a non-invasive technique for physiological and quantitative assessment of gastric emptying [41, 42]. Alternatives to GES include wireless motility capsules (WMC), stable-isotope gastric emptying breath testing (GEBT), and functional ultrasonography. However, additional data on their sensitivity and specificity are needed before they can be recommended for routine use [43-45].

\section{Management of GP recommended by the American Soci- ety of Gastroenterology (ASG)}

The ASG has put forth recommendations for the management of GP [46]. These strategies primarily focus on the correction of nutritional state, providing symptomatic relief, tighter glycemic control, and improvement of the gastric emptying times [46].

\section{Diet and nutritional support for GP}

Patients with GP may have calorie deficit due to poor oral intake, in addition to deficiencies of essential vitamins and minerals [47]. Therefore, in patients with mild disease, oral nutrition with small, low-fat, low-fibre meals about 4 - 5 times/ day is recommended. High calorie, nutritionally rich liquids are preferred over solids as gastric emptying of liquids is often normal in GP. Poor oral tolerance to liquids is indicative of poor outcomes [48]. Furthermore, oral intake of alcohol and tobacco should be avoided as they alter gastric emptying time $[49,50]$. In diabetics, good glycemic control and oral hypoglycemic agents can accelerate gastric emptying times. For those with severe GP or poor outcomes on oral nutrition, enteral or parenteral nutrition may be necessary. Enteral nutrition is preferred over parenteral nutrition for lower costs, potentially fewer complications, and ease of delivery. Enteral feeds may be started with diluted infusions and gradually advanced to iso-osmolar preparations at low infusion rates $(20 \mathrm{~mL} / \mathrm{h})$ which can then be gradually titrated to $60 \mathrm{~mL} / \mathrm{h}$ for $12-15 \mathrm{~h} /$ day to maintain adequate nutrition and hydration.

\section{Glycemic control in DGP}

In experimental studies, acute hyperglycemia has shown to delay gastric emptying and inhibit antral contractility. However, its exact association with clinical symptoms still is remains unclear. Additionally, the long-term effect of tight glycemic control on improvement of gastric emptying and resolution of symptoms is controversial. ASG recommends the use of dipeptidyl peptidase-4 (DPP-4) inhibitors such as sitagliptin and vildagliptin for glycemic control in DGP as these agents do not delay gastric emptying times [51].

\section{Pharmacotherapy for GP}

Prokinetic agents are recommended for symptomatic GP despite dietary modifications. The first line prokinetic agent is metoclopramide (dopamine-2 receptor antagonist), which enhances gastric emptying time by improving gastric antrum contractions and decreasing post-prandial fundus relaxation. According to recommendations by the US Food and Drug Administration (FDA), metoclopramide should be used for no longer than 12 weeks for GP unless patients have therapeutic benefits which outweigh the risk of therapy [52]. Domperidone, another agent approved for the treatment of GP, has similar efficacy as that of metoclopramide but fewer CNS side effects. It is used in patients who experience side effects or fail to improve on metoclopramide. Furthermore, patients who fail to respond to both these agents may candidates for eryth- 
romycin which acts on motilin receptors and promotes gastric emptying.

\section{Symptomatic management of nausea, vomiting and pain}

Antiemetic agents such as ondansetron, prochlorperazine, thiethylperazine, or antihistamines (promethazine) can be used for symptomatic nausea and vomiting. However, patients with refractory nausea, vomiting and abdominal pain may require low dose tricyclic antidepressants (TCA) [53].

\section{Gastric electrical stimulation (GES)}

The US FDA approved GES as a humanitarian device exemption for patients with refractory GP of idiopathic or diabetic origin [54]. Literature reports significant improvement in symptoms, quality of life and tolerance to oral feeds with the GES device [55]. However, additional controlled studies are still needed to estimate the actual benefit.

\section{Surgical intervention}

Surgical interventions are not routine and rarely indicated for GP. According to literature, venting gastrectomy has been associated with marked symptomatic improvement, maintenance of weight and reduction in the total symptom score 3 years post procedure [56]. It is assumed that percutaneous endoscopic gastrostomy is less invasive with similar benefits. Furthermore, surgical interventions such as gastrojejunostomy, pyloromyotomy, and completion or subtotal gastrectomy have been tried for GP; however, long-term follow-up studies are needed before these interventions can be recommended.

\section{Alternative therapies}

These include acupuncture and physical retraining modalities. Several studies have demonstrated the benefit of acupuncture in GP. A recent single-blinded, randomized pilot study with sham treatment control reported improvement in symptom severity and shorted gastric emptying to solids with electroacupuncture compared to baseline; however, the gastric emptying times in the active and sham-controlled arms were not matched [57]. Additionally, autonomic retraining techniques which were developed by NASA for space motion sickness have also shown some benefit [58].

GP is known to significantly decrease the quality of life. In literature, mortality rates for GP range from $4 \%$ to $38 \%$ with the highest reported mortality in diabetics requiring nutritional support $[59,60]$. Studies report better mortality outcomes for patients treated in outpatient settings after a 2-year followup [60]. A population-based study by Jung et al reported mortality for about one-third of the patients with GP and further went on to describe lower survival rates for GP compared to the general population [12]. In our study, the inpatient mortal- ity for GP was found to be $0.25 \%$. After adjusting for CCI, we report higher odds of mortality in the NDGP group compared to the DGP group despite DGP hospitalizations making up $78.1 \%$ of the study population. The reason for this variance in mortality is unknown; however, it can be postulated that an early diagnosis and treatment of DGP may have in part influenced the mortality rates. Additionally, patients with NDGP had higher mean length of hospital stay compared to the DGP group. The reduction in hospital stay for DGP may be secondary to early diagnosis and initiation of treatment. We also report a higher mean total healthcare cost for NDGP $(\$ 44,100)$ compared to DGP hospitalizations $(\$ 35,500)$, which could be explained by the fact that patients with NDGP may require additional investigations to identify the underlying etiology. However, it should be noted that this is a stark increase in the mean total healthcare cost from $\$ 28,135$ in 2006 for inpatient management of GP [61]. This data reflect an increasing burden of GP on the US healthcare system. Moreover, patient satisfaction for the treatment of GP continues to be a growing concern as majority of the patients are not satisfied with the treatment they receive [62]. Hence, we believe that there is need for urgent policy intervention for cost reduction and effective management of GP. Furthermore, patients with GP are at increased risk of complications. We report higher odds of sepsis, DVT and PE in the NDGP group compared to the DGP group. This may be because NDGP hospitalizations have longer length of hospital stay and hence, are more prone to developing inpatient system-based complications. Additionally, we report higher odds of AKI in DGP hospitalizations. This may be explained by the fact that severe vomiting, the most prominent symptom of DGP, may lead to dehydration and prerenal AKI.

\section{Strengths and limitations}

Like any retrospective study, this study has several strengths and limitations. The greatest strength of this study is the study population itself. The sample population used for the analysis is derived from one of the largest, publicly available, multiethnic hospital-based US registries developed through a FederalState-Industry partnership. It contains hospitalization data for nearly all inpatient admission and hence, the study is applicable to all hospitals across the US. Moreover, this study includes and examines numerous outcome-oriented facets and epidemiology of GP hospitalizations. This allows for an extremely comprehensive overview of GP admissions. However, as with any study, there are bound to be limitations. The NIS database does not contain information for the severity of the disease and time of diagnosis. Additionally, we were unable to analyze the outcome of GP with liver disease as this analysis would fall outside the scope of our study. Furthermore, data gathered from NIS may be subject to biases present in retrospective studies. The hospitalizations identified for the study were not of individual patients but of GP hospitalizations. Hence, people admitted numerous times for the same complaints would be included several times within the data set. Finally, NIS is an administrative database that uses a coding system to gather clinical outcomes and information; therefore, a possibility 
of coding errors cannot be ruled out. However, despite these limitations, we believe that the large sample size, study design, analysis technique and answers to scientific questions contribute to a better understanding of GP. This study aims to not only stimulate intellectual conversations on the topic at hand, but also to encourage large multicenter prospective studies on GP.

\section{Conclusions}

GP is a chronic medical condition characterized by delayed or absent gastric emptying in the absence of a mechanical obstruction. It is a relatively under-investigated disease pathology, and the exact incidence and prevalence in the general population is currently unknown. However, over the past few decades, it has grabbed the interest of investigators. The two most common etiologies of GP reported in literature include idiopathic causes and diabetes mellitus. Compared to patients with DGP, we noted a higher percentage of women, Whites, patients with a history of smoking, and COPD in the NDGP group. Furthermore, the DGP group had higher proportions of Blacks, Hispanics, and patient with hypertension, obesity, congestive heart failure and chronic kidney disease. GP may present with a wide range of clinical symptoms such as nausea, vomiting, abdominal pain, postprandial fullness, early satiety, bloating, and loss of weight and apatite. In patients with suspected GP, a thorough history and physical examination are essential to identify the underlying cause or associated factors. Gastric emptying scintigraphy is the gold standard investigation to assess gastric emptying in patients where mechanical obstruction has been ruled out via other radiological investigations. GP is associated with significant morbidity and mortality. In our study, the inpatient mortality for GP was $0.25 \%$. However, compared to GP hospitalizations, patients with NDGP had higher odds of inpatient mortality, and were associated with a longer length of hospital stay, mean total healthcare cost and higher odds of system-based complications such as sepsis, DVT and PE.

\section{Acknowledgments}

None to declare.

\section{Financial Disclosure}

The authors have received no funding with respect to research, authorship, and/or publication of this article.

\section{Conflict of Interest}

The authors report no conflict of interest.

\section{Informed Consent}

Not applicable.

\section{Author Contributions}

Hafeez Shaka and Asim Kichloo are credited with substantial contribution to the design of the work, acquisition and interpretation of the data, drafting the manuscript, statistical analysis, revision of important intellectual content, final approval of the version published, and agreement of accountability for all aspects of the work. Dushyant Sign Dahiya and Farah Wani are credited with substantial contribution to interpretation of data, literature review of all sections discussed, drafting of the manuscript, final approval of the version published, and agreement of accountability for all aspects of the work. Jagmeet Singh, Ehizogie Edigin, Asad Mehboob and Micheal Albosta are credited with interpretation of the data, literature review of all sections, revision of important intellectual content, final approval of the version published, and agreement of accountability of all aspects of the work.

\section{Data Availability}

We used and/or analyzed the NIS database for 2016 and 2017, available online at http: //www.hcup- us.ahrq.gov. The NIS is a large publicly available all-payer inpatient care database in the USA, containing data on more than 7 million hospital stays yearly. Its large sample size is ideal for developing national and regional estimates and enables analyses of rare conditions, uncommon treatments, and special populations.

\section{Abbreviations}

GP: gastroparesis; DGP: diabetic gastroparesis; NDGP: nondiabetic gastroparesis; NIS: Nationwide Inpatient Sample; CCI: Charlson comorbidity index; DVT: deep vein thrombosis; PE: pulmonary embolism; AKI: acute kidney injury; ICD10: International Classification of Diseases, Tenth Revision, Clinical Modification/Procedure Coding System; LOS: length of stay; THC: total hospital charges; PSGP: post-surgical gastroparesis; PVGP: post-viral gastroparesis; CMV: cytomegalovirus; EBV: Epstein-Barr virus; VZV: varicella-zoster virus; CNS: central nervous system; GAD: glutamic acid decarboxylase; COPD: chronic obstructive pulmonary disease; $\mathrm{CT}$ : computed tomography; MRI: magnetic resonance imaging; GES: gastric emptying scintigraphy; WMC: wireless motility capsule; GEBT: stable-isotope gastric emptying breath testing; ASG: American Society of Gastroenterology; FDA: Food and Drug Administration; TCA: tricyclic antidepressants; DPP-4: dipeptidyl peptidase-4

\section{References}

1. Usai-Satta P, Bellini M, Morelli O, Geri F, Lai M, Bassotti G. Gastroparesis: new insights into an old disease. World J Gastroenterol. 2020;26(19):2333-2348.

2. Stanghellini V, Tack J. Gastroparesis: separate entity or 
just a part of dyspepsia? Gut. 2014;63(12):1972-1978.

3. Parkman HP, Fass R, Foxx-Orenstein AE. Treatment of patients with diabetic gastroparesis. Gastroenterol Hepatol (N Y). 2010;6(6):1-16.

4. Baumgardner DJ. Suffering in silence: is gastroparesis underdiagnosed? J Patient Cent Res Rev. 2019;6(2):133134.

5. Hasler WL. Gastroparesis - current concepts and considerations. Medscape J Med. 2008;10(1):16.

6. Healthcare Cost and Utilization Project (HCUP). Overview of the National (Nationwide) Inpatient Sample (NIS). https://www.hcup-us.ahrq.gov/nisoverview.jsp. Accessed November 1st, 2020.

7. Healthcare cost and utilization Project (HCUP). NIS Database Documentation. https://www.hcup-us.ahrq.gov/ $\mathrm{db} /$ nation/nis/nisdbdocumentation.jsp. Accessed November 1 st, 2020.

8. Germanovskii, II, Astakhova EI. [Roentgenologic investigation on the gastric evacuant function in gastroparesis]. Klin Med (Mosk). 1951;29(3):35-41.

9. Grover M, Farrugia G, Stanghellini V. Gastroparesis: a turning point in understanding and treatment. Gut. 2019;68(12):2238-2250.

10. Parkman HP. Idiopathic gastroparesis. Gastroenterol Clin North Am. 2015;44(1):59-68.

11. Wadhwa V, Mehta D, Jobanputra Y, Lopez R, Thota PN, Sanaka MR. Healthcare utilization and costs associated with gastroparesis. World J Gastroenterol. 2017;23(24):44284436.

12. Jung HK, Choung RS, Locke GR, 3rd, Schleck CD, Zinsmeister AR, Szarka LA, Mullan B, et al. The incidence, prevalence, and outcomes of patients with gastroparesis in Olmsted County, Minnesota, from 1996 to 2006. Gastroenterology. 2009;136(4):1225-1233.

13. Rey E, Choung RS, Schleck CD, Zinsmeister AR, Talley NJ, Locke GR, 3rd. Prevalence of hidden gastroparesis in the community: the gastroparesis "iceberg". J Neurogastroenterol Motil. 2012;18(1):34-42.

14. Spanakis EK, Golden SH. Race/ethnic difference in diabetes and diabetic complications. Curr Diab Rep. 2013;13(6):814-823.

15. American Diabetes Association. Diagnosis and classification of diabetes mellitus. Diabetes Care. 2010;33(Suppl 1):S62-69.

16. Vittal H, Farrugia G, Gomez G, Pasricha PJ. Mechanisms of disease: the pathological basis of gastroparesis - a review of experimental and clinical studies. Nat Clin Pract Gastroenterol Hepatol. 2007;4(6):336-346.

17. Koch KL. Diabetic gastropathy: gastric neuromuscular dysfunction in diabetes mellitus: a review of symptoms, pathophysiology, and treatment. Dig Dis Sci. 1999;44(6):1061-1075.

18. Krishnasamy S, Abell TL. Diabetic gastroparesis: principles and current trends in management. Diabetes Ther. 2018;9(Suppl 1):1-42.

19. Shafi MA, Pasricha PJ. Post-surgical and obstructive gastroparesis. Curr Gastroenterol Rep. 2007;9(4):280-285.

20. Nassar Y, Richter S. Gastroparesis in non-diabetics: associated conditions and possible risk factors. Gastroenterol- ogy Res. 2018;11(5):340-345.

21. Sigurdsson L, Flores A, Putnam PE, Hyman PE, Di Lorenzo C. Postviral gastroparesis: presentation, treatment, and outcome. J Pediatr. 1997;131(5):751-754.

22. Meeroff JC, Schreiber DS, Trier JS, Blacklow NR. Abnormal gastric motor function in viral gastroenteritis. Ann Intern Med. 1980;92(3):370-373.

23. Vassallo M, Camilleri M, Caron BL, Low PA. Gastrointestinal motor dysfunction in acquired selective cholinergic dysautonomia associated with infectious mononucleosis. Gastroenterology. 1991;100(1):252-258.

24. Lobrano A, Blanchard K, Abell TL, Minocha A, Boone W, Wyatt-Ashmead J, Fratkin J, et al. Postinfectious gastroparesis related to autonomic failure: a case report. Neurogastroenterol Motil. 2006;18(2):162-167.

25. Naftali T, Yishai R, Zangen T, Levine A. Post-infectious gastroparesis: clinical and electerogastrographic aspects. J Gastroenterol Hepatol. 2007;22(9):1423-1428.

26. Thongpooswan S, Chyn E, Alfishawy M, Restrepo E, Berman C, Ahmed K, Muralidharan S. Polyradiculopathy and gastroparesis due to cytomegalovirus infection in AIDS: a case report and review of literature. Am J Case Rep. 2015;16:801-804.

27. Reddivari AKR, Mehta P: Gastroparesis. In: StatPearls. Treasure Island (FL), 2020.

28. Quigley EM. Other forms of gastroparesis: postsurgical, Parkinson, other neurologic diseases, connective tissue disorders. Gastroenterol Clin North Am. 2015;44(1):6981.

29. Mukherjee A, Biswas A, Das SK. Gut dysfunction in Parkinson's disease. World J Gastroenterol. 2016;22(25):57425752.

30. Chow D, Nakamoto BK, Sullivan K, Sletten DM, Fujii S, Umekawa S, Kocher M, et al. Symptoms of Autonomic Dysfunction in Human Immunodeficiency Virus. Open Forum Infect Dis. 2015;2(3):ofv103.

31. Soota K, Kedar A, Nikitina Y, Arendale E, Vedanarayanan V, Abell TL. Immunomodulation for treatment of drug and device refractory gastroparesis. Results Immunol. 2016;6:11-14.

32. Dhamija R, Tan KM, Pittock SJ, Foxx-Orenstein A, Benarroch E, Lennon VA. Serologic profiles aiding the diagnosis of autoimmune gastrointestinal dysmotility. Clin Gastroenterol Hepatol. 2008;6(9):988-992.

33. Dalakas MC. Intravenous immunoglobulin in patients with anti-GAD antibody-associated neurological diseases and patients with inflammatory myopathies: effects on clinicopathological features and immunoregulatory genes. Clin Rev Allergy Immunol. 2005;29(3):255-269.

34. Clark MB, Davis T. A pediatric case of severe pandysautonomia responsive to plasmapheresis. J Child Neurol. 2013;28(12):1716-1719.

35. Camilleri M, Chedid V, Ford AC, Haruma K, Horowitz M, Jones KL, Low PA, et al. Gastroparesis. Nat Rev Dis Primers. 2018;4(1):41.

36. Weston S, Thumshirn M, Wiste J, Camilleri M. Clinical and upper gastrointestinal motility features in systemic sclerosis and related disorders. Am J Gastroenterol. 1998;93(7):1085-1089. 
37. Parkman HP, Yates K, Hasler WL, Nguyen L, Pasricha PJ, Snape WJ, Farrugia G, et al. Similarities and differences between diabetic and idiopathic gastroparesis. Clin Gastroenterol Hepatol. 2011;9(12):1056-1064; quiz e1133-1054.

38. Hoogerwerf WA, Pasricha PJ, Kalloo AN, Schuster MM. Pain: the overlooked symptom in gastroparesis. Am J Gastroenterol. 1999;94(4):1029-1033.

39. Hasler WL, Wilson LA, Parkman HP, Nguyen L, Abell TL, Koch KL, Pasricha PJ, et al. Bloating in gastroparesis: severity, impact, and associated factors. Am J Gastroenterol. 2011;106(8):1492-1502.

40. Liu N, Abell T. Gastroparesis updates on pathogenesis and management. Gut Liver. 2017;11(5):579-589.

41. Shin AS, Camilleri M. Diagnostic assessment of diabetic gastroparesis. Diabetes. 2013;62(8):2667-2673.

42. Abell TL, Camilleri M, Donohoe K, Hasler WL, Lin HC, Maurer AH, McCallum RW, et al. Consensus recommendations for gastric emptying scintigraphy: a joint report of the American Neurogastroenterology and Motility Society and the Society of Nuclear Medicine. Am J Gastroenterol. 2008;103(3):753-763.

43. Kuo B, McCallum RW, Koch KL, Sitrin MD, Wo JM, Chey WD, Hasler WL, et al. Comparison of gastric emptying of a nondigestible capsule to a radio-labelled meal in healthy and gastroparetic subjects. Aliment Pharmacol Ther. 2008;27(2):186-196.

44. Agency for Healthcare Research and Quality (AHRQ). Wireless motility capsule versus other diagnostic technologies for evaluating gastroparesis and constipation: A comparative effectiveness review (No. 110). Available at: http:// effectivehealthcare.ahrq.gov/ehc/products/392/1498/Constipation-gastroparesis-wireless-capsule-report-130520. pdf. Accessed November 15th, 2020.

45. Szarka LA, Camilleri M, Vella A, Burton D, Baxter K, Simonson J, Zinsmeister AR. A stable isotope breath test with a standard meal for abnormal gastric emptying of solids in the clinic and in research. Clin Gastroenterol Hepatol. 2008;6(6):635-643 e631.

46. Camilleri M, Parkman HP, Shafi MA, Abell TL, Gerson L, American College of G. Clinical guideline: management of gastroparesis. Am J Gastroenterol. 2013;108(1):18-37; quiz 38.

47. Parkman HP, Yates KP, Hasler WL, Nguyan L, Pasricha PJ, Snape WJ, Farrugia G, et al. Dietary intake and nutritional deficiencies in patients with diabetic or idiopathic gastroparesis. Gastroenterology. 2011;141(2):486-498, 498 e481-487.

48. Abell TL, Bernstein RK, Cutts T, Farrugia G, Forster J, Hasler WL, McCallum RW, et al. Treatment of gastroparesis: a multidisciplinary clinical review. Neurogastroen- terol Motil. 2006;18(4):263-283.

49. Pfeiffer A, Hogl B, Kaess H. Effect of ethanol and commonly ingested alcoholic beverages on gastric emptying and gastrointestinal transit. Clin Investig. 1992;70(6):487491.

50. Scott AM, Kellow JE, Eckersley GM, Nolan JM, Jones MP. Cigarette smoking and nicotine delay postprandial mouthcecum transit time. Dig Dis Sci. 1992;37(10):1544-1547.

51. Vella A, Bock G, Giesler PD, Burton DB, Serra DB, Saylan ML, Dunning BE, et al. Effects of dipeptidyl peptidase-4 inhibition on gastrointestinal function, meal appearance, and glucose metabolism in type 2 diabetes. Diabetes. 2007;56(5):1475-1480.

52. Available from: http://www.fda.gov/newsevents/newsroom /pressannouncements/ucm149533.htm. Accessed November 11th 2020.

53. Prakash C, Lustman PJ, Freedland KE, Clouse RE. Tricyclic antidepressants for functional nausea and vomiting: clinical outcome in 37 patients. Dig Dis Sci. 1998;43(9):1951-1956.

54. Humanitarian device exemption for Enterra device. Federal Registry. 2000;65:78495-78496.

55. Abell TL, Johnson WD, Kedar A, Runnels JM, Thompson J, Weeks ES, Minocha A, et al. A double-masked, randomized, placebo-controlled trial of temporary endoscopic mucosal gastric electrical stimulation for gastroparesis. Gastrointest Endosc. 2011;74(3):496-503 e493.

56. Kim $\mathrm{CH}$, Nelson DK. Venting percutaneous gastrostomy in the treatment of refractory idiopathic gastroparesis. Gastrointest Endosc. 1998;47(1):67-70.

57. Wang CP, Kao CH, Chen WK, Lo WY, Hsieh CL. A single-blinded, randomized pilot study evaluating effects of electroacupuncture in diabetic patients with symptoms suggestive of gastroparesis. J Altern Complement Med. 2008;14(7):833-839.

58. Rashed H, Cutts T, Abell T, Cowings P, Toscano W, ElGammal A, Adl D. Predictors of response to a behavioral treatment in patients with chronic gastric motility disorders. Dig Dis Sci. 2002;47(5):1020-1026.

59. Bielefeldt K. Gastroparesis: concepts, controversies, and challenges. Scientifica (Cairo). 2012;2012:424802.

60. Bharucha AE. Epidemiology and natural history of gastroparesis. Gastroenterol Clin North Am. 2015;44(1):9-19.

61. Hirsch W, Nee J, Ballou S, Petersen T, Friedlander D, Lee HN, Cheng V, et al. Emergency department burden of gastroparesis in the United States, 2006 to 2013. J Clin Gastroenterol. 2019;53(2):109-113.

62. Yu D, Ramsey FV, Norton WF, Norton N, Schneck S, Gaetano T, Parkman HP. The burdens, concerns, and quality of life of patients with gastroparesis. Dig Dis Sci. 2017;62(4):879-893. 